\title{
Robust temporal alignment of multimodal cardiac sequences
}

\author{
Andrea Perissinotto $^{a}$, Sandro Queirós ${ }^{a, b, c}$, Pedro Morais ${ }^{a}$, Maria J. Baptista ${ }^{a}$, Mark \\ Monaghan $^{d}$, Nuno F. Rodrigues ${ }^{a, c, e}$, Jan D'hooge ${ }^{b}$, João L. Vilaça ${ }^{a, e}$, Daniel Barbosa ${ }^{a, e}$ \\ ${ }^{a}$ ICVS/3B's - PT Government Associate Laboratory, Braga/Guimarães, Portugal \\ ${ }^{b}$ Lab on Cardiovascular Imaging and Dynamics, KU Leuven, Belgium \\ ${ }^{c}$ Algoritmi Center, School of Engineering, University of Minho, Guimarães, Portugal \\ ${ }^{d}$ Department of Cardiology, King's College Hospital, London, United Kingdom \\ ${ }^{e}$ DIGARC - Polytechnic Institute of Cávado and Ave, Barcelos, Portugal
}

\begin{abstract}
Given the dynamic nature of cardiac function, correct temporal alignment of pre-operative models and intraoperative images is crucial for augmented reality in cardiac image-guided interventions. As such, the current study focuses on the development of an image-based strategy for temporal alignment of multimodal cardiac imaging sequences, such as cine Magnetic Resonance Imaging (MRI) or 3D Ultrasound (US). First, we derive a robust, modality-independent signal from the image sequences, estimated by computing the normalized crosscorrelation between each frame in the temporal sequence and the end-diastolic frame. This signal is a resembler for the left-ventricle (LV) volume curve over time, whose variation indicates different temporal landmarks of the cardiac cycle. We then perform the temporal alignment of these surrogate signals derived from MRI and US sequences of the same patient through Dynamic Time Warping (DTW), allowing to synchronize both sequences. The proposed framework was evaluated in 98 patients, which have undergone both 3D+t MRI and US scans. The end-systolic frame could be accurately estimated as the minimum of the image-derived surrogate signal, presenting a relative error of $1.6 \pm 1.9 \%$ and $4.0 \pm 4.2 \%$ for the MRI and US sequences, respectively, thus supporting its association with key temporal instants of the cardiac cycle. The use of DTW reduces the desynchronization of the cardiac events in MRI and US sequences, allowing to temporally align multimodal cardiac imaging sequences. Overall, a generic, fast and accurate method for temporal synchronization of MRI and US sequences of the same patient was introduced. This approach could be straightforwardly used for the correct temporal alignment of pre-operative MRI information and intra-operative US images.
\end{abstract}

Keywords: Temporal alignment, multimodal cardiac image sequences, dynamic time warping.

\section{INTRODUCTION}

A significant amount of cardiovascular diseases, the world's leading cause of death, require surgical or interventional treatment. In recent years, the development of cardiac minimally invasive (MI) procedures has drastically decreased the number of open-heart surgeries, which are related to complications such as embolism and stroke. MI interventions are strictly connected to image guidance systems, which allow the physician to have a detailed knowledge on both cardiac anatomy and the 3D position of surgical instrumentation. Recently, cardiac 3D imaging modalities for interventional image guidance have been improved, introducing the ability for 4D (3D $+\mathrm{t})$ sequence acquisitions. ${ }^{1}$ Such $4 \mathrm{D}$ imaging allows to obtain real-time information of the beating heart, but also improves the surgical view by depicting the 3D position of the surgical instruments in real-time. Among intraoperative imaging modalities, real-time 3D echocardiography (RT3DE) offers important advantages in terms of temporal resolution and portability, ${ }^{2}$ while remaining an approach free of ionizing radiation, thus being safe to both the patient and the operating physician. ${ }^{3}$ Nevertheless, the self-standing use of RT3DE imaging is inadequate for guidance purpose, since it offers only moderate spatial resolution and signal to noise ratio (SNR), impairing the identification of small anatomical details. ${ }^{1}$ A valid solution to such a problem consists in drawing benefits from different image modalities introducing the superposition of high quality pre-operative models, such as cine magnetic resonance imaging (MRI), onto real-time intra-operative images. ${ }^{4}$ Nonetheless, due to the introduction of temporal variability between $4 \mathrm{D}$ sequences, image registration has proven to be a very complex problem. Therefore, it is essential to perform a temporal alignment between sequences, synchronizing the main

Medical Imaging 2015: Image Processing, edited by Sébastien Ourselin, Martin A. Styner, Proc. of SPIE Vol. 9413, 94131K · (C) 2015 SPIE · CCC code: 1605-7422/15/\$18 · doi: 10.1117/12.2082712 
stages of the cardiac cycle, discerned through the identification of temporal landmarks. However, the different modalities employed to acquire the sequences have different image appearance, introducing an additional obstacle in identifying common temporal reference points.

Several authors approach the problem of cardiac temporal registration, handling the temporal variability of the heart through an electrocardiography (ECG)-gated image acquisition. For instance, the classic cardiac cineMRI scan, is performed restricting the acquisition of the same 2D slice in a fixed number of time points of the cycle, identified on the ECG signal. Small spatial increments in acquisition coordinates, orthogonally directed to the image plane, allow to reconstruct the entire $3 \mathrm{D}$ volume. ${ }^{5}$ As a consequence, after several cycles, 3D images at distinct time points are available, simplifying temporal alignment, but preventing the use of this protocol for real-time applications. Peyrat et al. ${ }^{6}$ extended this process for real-time application, extracting 3D-CT frames at a defined percentage of the cardiac cycle (R-R interval), identified through an ECG-gated acquisition. Then, a characteristic blood volume curve allowed to identify time points associated to the mechanical state of the heart, which led to a non-linear transformation, able to identify the optimal match between correspondent frames. Nevertheless, in this framework, the temporal registration is strictly dependent on a controlled acquisition protocol, likewise performed in both sequences. In absence of such protocol, the temporal match of cardiac phases relies on a transformation able to warp sequences, pairing frames depicting consistent events in the two sequences.

In literature, many other frameworks also employ ECG-based strategies, ensuring that the acquisition includes a complete cardiac cycle in which the first frame coincides with end-diastole. Huang et al. ${ }^{7}$ noticed that the different acquisition rates of ECG-gated US and MRI/CT sequences was the main responsible for temporal mismatching. Therefore, for each US image, the corresponding frame in MRI/CT was computed through linear interpolation of this high quality sequence, obtaining a complete temporal correspondence between frames. Nonetheless, their method does not provide a solution to the intrinsic variability between consecutive cardiac cycles, associated to the constant adaptation of the heart to the varying physiological conditions of blood demand. These variations in heart rate cannot be compensated by direct rescaling of the temporal space, since a higher contracting rate is primarily associated to a decrease in the duration of the diastolic stage of the cycle and is not uniformly changing the cardiac cycle. In other words, when a reduction of the pulsation occurs, there is a larger reduction of the duration of diastole then of the systolic one. ${ }^{8}$ Shekar et al. ${ }^{9}$ proposed to compensate this physiological effect by asking the user to manually indicate the end-diastolic (ED) and end-systolic (ES) timing in pre- and post-stress ECG traces. This information was then used to apply independent piece-wise temporal scaling to the systolic and diastolic phases of the image sequences. Nonetheless, this method guarantees the alignment of only two time points (ES and ED) for each cycle, while the piece-wise scaling could introduce undesired pairing of the remaining cardiac stages. Moreover, this approach remains user-dependent and the accuracy of the key frame detection is intrinsically related to the user experience. Finally, the ECG trace can be distorted between acquisitions for several reasons, including the strong MRI magnetic field. ${ }^{10}$

The methodologies mentioned so far require additional information (ECG, user steering, blood volume curve) as reference for the alignment, which result in additional data, vulnerable to distortions, which is required to be stored during acquisitions. Therefore, the introduction of completely image-based methodologies avoid to acquire such additional data, since the information required for temporal alignment is included in the image sequence itself.

In this direction, Perperidis et al. ${ }^{10}$ achieved the alignment of $4 \mathrm{D}$ cardiac MRI sequences applying a temporal transformation, consisting in a global part and in a local part. Global features, such as difference in cycle length or in frame rate of the acquisitions, were addressed by a global temporal transformation, while differences in the length of each cardiac stage were corrected by local transformations. The local transformation, modeled using a B-spline, relied on the identification, on both sequences, of four relevant cardiac cycle points: start of the cycle, maximum contraction (end-systole), end-diastole and end of the cycle. These key stages were identified through a completely image-based cross-correlation curve. In detail, the image cross-correlation was computed between each 3D frame in the sequence with the first frame, which corresponds to the start of the cardiac cycle. The resulting curve allowed to easily extract relevant points: the end-systolic and the end-diastolic time positions. As a result, the two sequences were temporally aligned overlapping the first and last frame (start and end of cardiac cycle) and the two extracted time landmarks. 
Zhang et al. ${ }^{11}$ used a similar approach to align $2 \mathrm{D}+\mathrm{t}$ MRI and US sequences. The alignment process relied initially on the identification of key-stages (initial time point, end-systolic volume time point, end-diastolic volume time point and final time point) through the cross-correlation signal. Once this was achieved, it was ensured that the two sequences contained the same number of frames, aligning the key-frames in the two sequences and interpolating, if necessary, the shorter sequence between those points. As result, the two sequences have the same amount of frames, generating complete temporal correspondence.

The main goal of the present study was to perform real-time alignment of dynamic pre-operative high quality MR data with dynamic intra-operative real-time 3D echocardiography (RT3DE) volumetric images of the beating heart. To this end, we aim to rely solely on image-based information to improve the robustness of the proposed solution against variations in the surgical theater. Firstly, we extract the image-based cross-correlation curve, whose trend marks the key-stages of heart activity, generating a modality-independent temporal reference. The next step consists in identifying an algorithm to match the content of these curves obtaining the temporal alignment of cardiac stages. To this end, instead of aligning a set of temporal landmarks, we will take the entire cycle into consideration. Wang et al. ${ }^{12}$ adopted the Dynamic Time Warping (DTW) to align generic curves over time. Raghavendra et al. ${ }^{13}$ introduced the algorithm in the biomedical field, as a distance measure to compare arrhythmic beats in an ECG real-time acquisition with normal ECG beats. In this paper, we employ the DTW to warp the correlation-based signals, finding the optimal alignment between them. Such temporal alignment tool will allow not only synchronized visualization of pre and intra-operative data, which can be used as a first step towards augmented reality frameworks where the high quality models derived from pre-operative data are used, but it could be equally employed in the construction of 4D statistical shape and motion models, which require a consistent temporal alignment of the organ undergoing a dynamic process.

\section{METHODS}

Since temporal alignment via traditional image registration methods is a computationally time-consuming strategy, ${ }^{7}$ we have chosen to follow an alternative image-based strategy. First, we derive a robust, modalityindependent signal from each image sequence. Such curve replaces the need of segmenting any anatomical structure, encoding the different phases of the cardiac cycle as temporal image decorrelation is associated with motion of cardiac structures. Once such signal is available for both MRI and RT3DE sequences of the same patient, we perform the temporal alignment of their content, synchronizing the key temporal moments of the cardiac cycle. After its application, the last step consists in transferring the synchronization information from the temporally aligned correlation curves to the original MR and RT3DE sequences, thus enabling their synchronization.

\subsection{Robust image-based surrogates for cardiac cycle characterization}

Kachenoura et al. ${ }^{14}$ proposed an image-based technique to define a signal whose features allow to identify essential events in the cardiac cycle. For each time sample (corresponding to a frame in the sequence), a scalar is obtained by estimating the normalized cross-correlation coefficient $(r)$ between two frames: one corresponding to the time sample above-mentioned and the other corresponding to the end-diastolic frame, as:

$$
r(t)=\frac{\sum_{i} \sum_{j} \sum_{k}[I(i, j, k, t)-\bar{I}]\left[I_{E D}(i, j, k)-\bar{I}_{E D}\right]}{\sqrt{\sum_{i} \sum_{j} \sum_{k}[I(i, j, k, t)-\bar{I}]^{2}} \times \sqrt{\sum_{i} \sum_{j} \sum_{k}\left[I_{E D}(i, j, k)-\bar{I}_{E D}\right]^{2}}}
$$

where $I(i, j, k, t)$ is the image intensity of the frame $t$ at position $(i, j, k)$, and $I_{E D}(i, j, k)$ is the intensity of the end-diastolic frame at the same position. $\bar{I}$ and $\bar{I}_{E D}$ are the mean intensity values of $I$ and $I_{E D}$, respectively. The correlation signal $r$ can be thought as a surrogate for the volume curve of the LV cavity over time, whose variation clearly indicates different temporal phases of the cardiac cycle, such as the diastasis period. Furthermore, its minimum has been previously associated with the end-systolic frame. ${ }^{14}$ Moreover, the methodology remains modality-independent, since its key characteristics do not depend on the underlying image appearance, as it can be observed in Figure 1. Besides information regarding the end-systolic frame, and the extension towards its use to temporally synchronize multimodal cardiac data described in the current work, this signal also encodes global cardiac function information. Indeed, Afshin et al. ${ }^{15}$ showed that left ventricular ejection fraction in MRI data can be accurately recovered from these correlation-based signals using neural networks. 

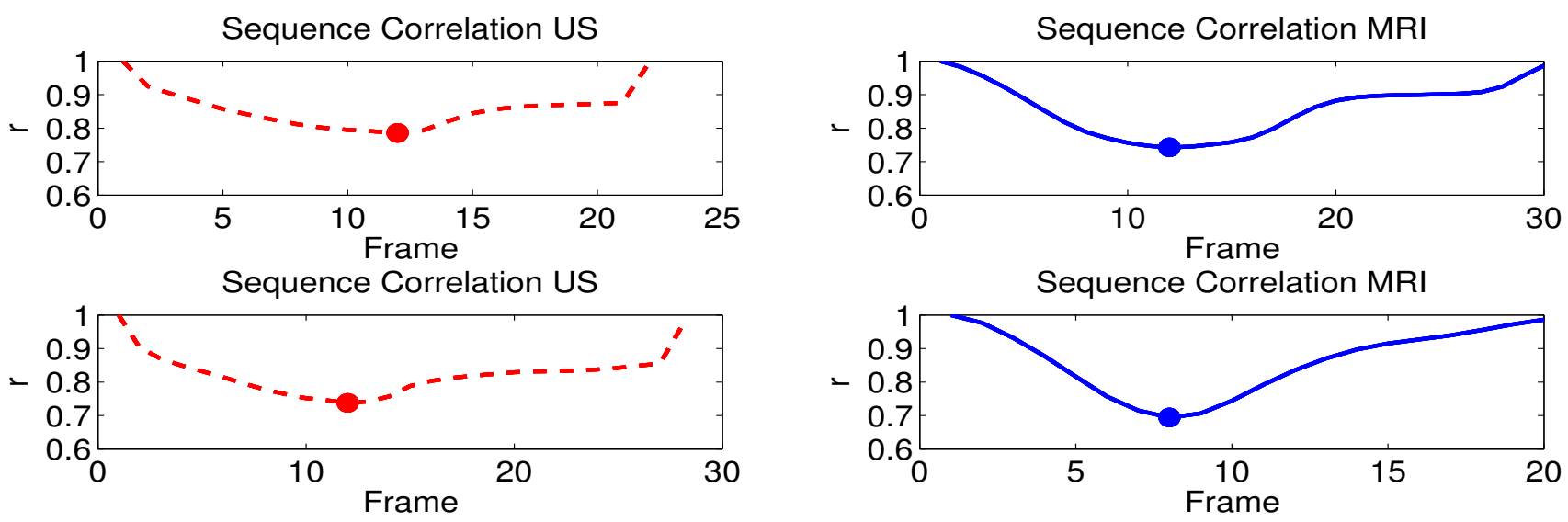

Sequence Correlation US
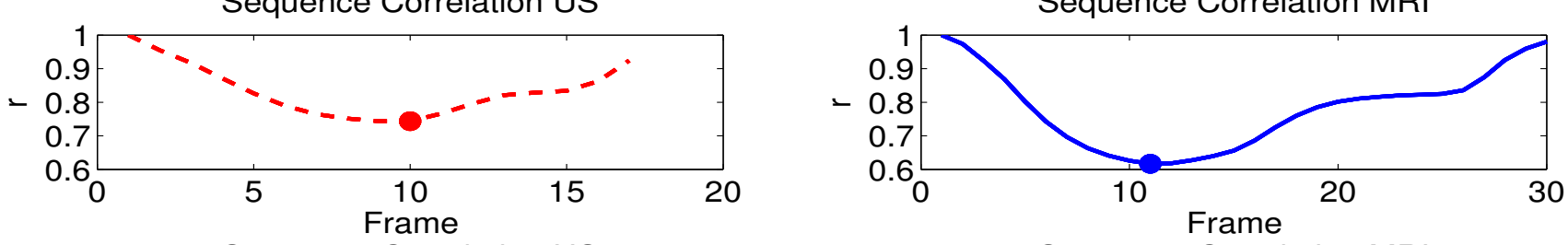

Sequence Correlation US
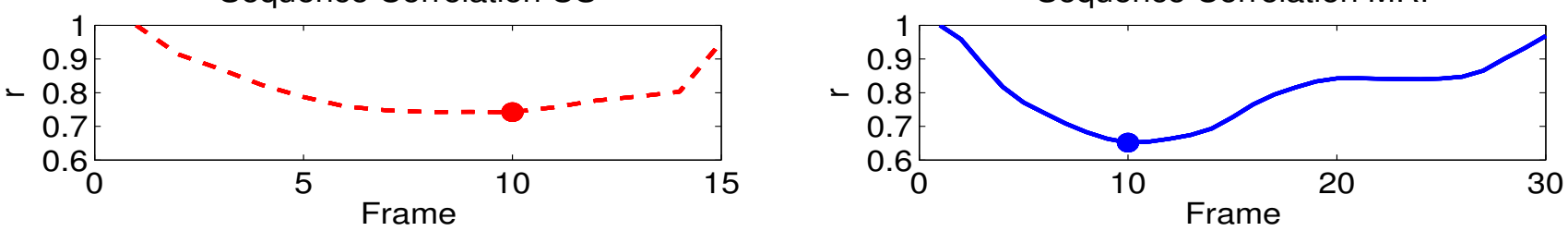

Figure 1: Correlation-based signals, $r$, obtained in four different patients for both 3D-US (red, left side) and MRI (blue, right side). Note that not only the image sequences have different number of frames in both modalities, but also the position of the end-systolic frame (red/blue dot) occurs at different relative instants between both scans.

\subsection{Temporal alignment of multimodal cardiac sequences}

The two computed correlation signals are initially pre-processed to compensate for potential differences in acquisition rate of both MRI and RT3DE sequences. As a consequence, the temporal axis is normalized employing a linear interpolation algorithm, in order to define two signals characterized by the same amount of samples $\left(N_{S}=100\right)$, thus compensating the frame disparity. The correlation-based signals are then normalized between 0 and 1 , which allows to compensate for different minimum values of $r$. An example of the resulting signals after this initial normalization step are shown in Figure 2a.

The Dynamic Time Warping (DTW) is applied to match the two normalized correlation-based signals generated for the MRI and US sequences of the same patient, with the purpose of estimating the optimal temporal alignment of these image sequences. DTW initially compares the paired differences between the correlation signals, $r_{M R I}$ and $r_{U S}$, which are discrete signals of same length $N_{S}$ due to the temporal axis normalization. The result is a $N_{S} \times N_{S}$ cost matrix (c) defined as:

$$
c\left(t_{M R I}, t_{U S}\right)=\left[r_{M R I}\left(t_{M R I}\right)-r_{U S}\left(t_{U S}\right)\right]^{2}
$$

where $r_{M R I}\left(t_{M R I}\right)$ and $r_{U S}\left(t_{U S}\right)$ are the correlation-based signals of the two sequences (MRI and US) evaluated respectively at $t_{M R I}$ and $t_{U S}$. This matrix establish an index of similarity between samples belonging to different sequences. The optimal alignment between $r_{M R I}$ and $r_{U S}$ can be recovered as finding an optimal path along the cost matrix $c\left(t_{M R I}, t_{U S}\right)$, as illustrated in Figure 2b. Consider a path $w=\left(w_{1} \ldots w_{l} \ldots w_{L}\right)$, where $w_{l}=$ $\left(t_{M R I_{l}}, t_{U S_{l}}\right)$ defines an element in $c\left(t_{M R I}, t_{U S}\right)$. The path $w$ represents a possible temporal alignment between $r_{M R I}$ and $r_{U S}$ if it satisfies the following three conditions: 


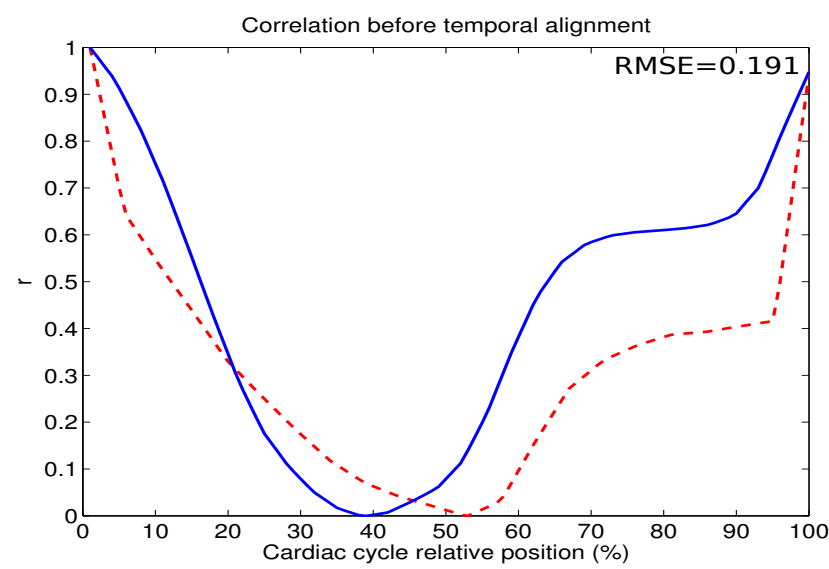

(a)

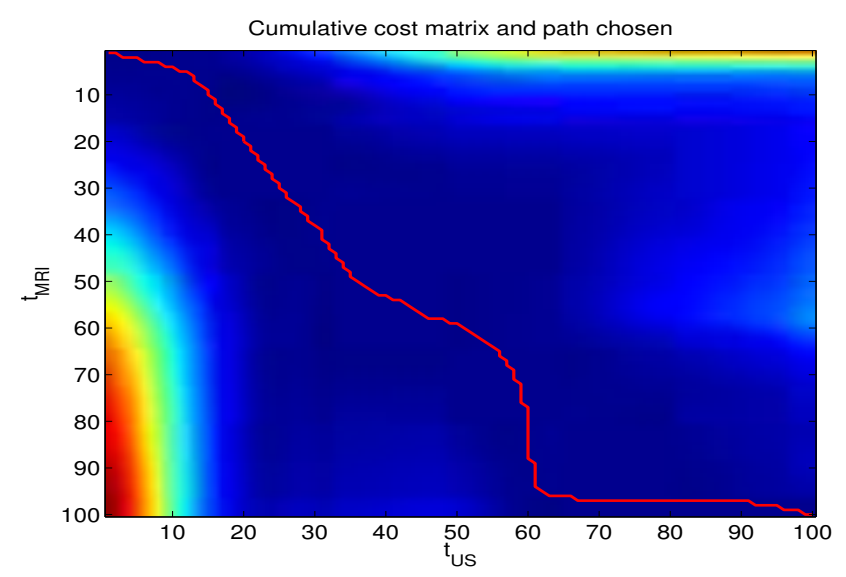

(c)

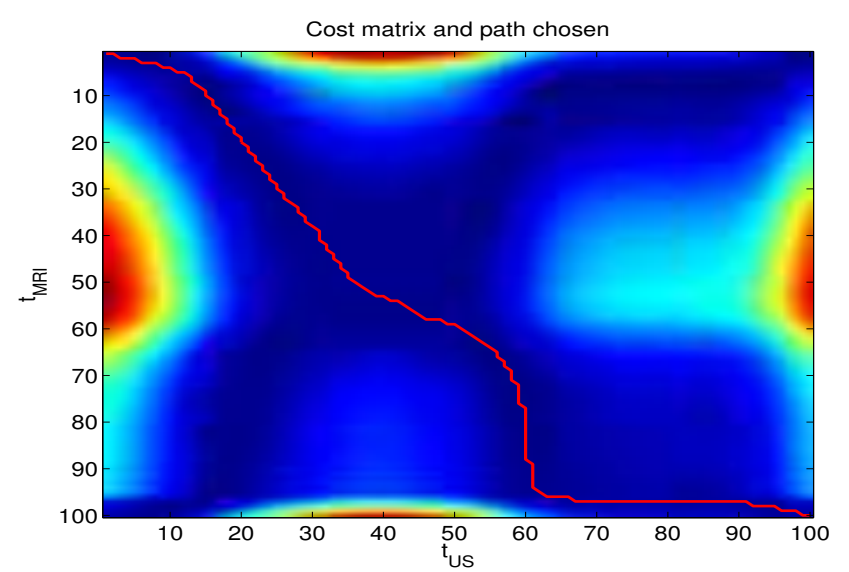

(b)

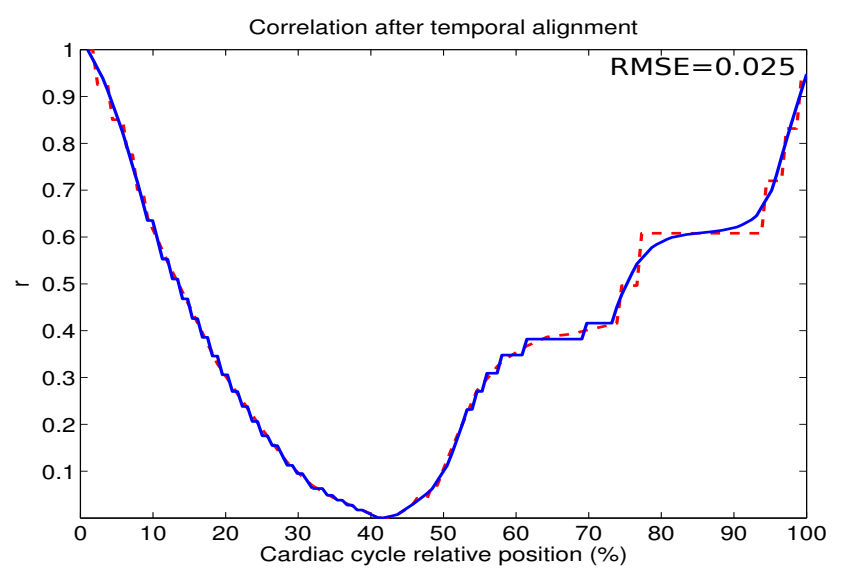

(d)

Figure 2: (a) Correlation after normalization and interpolation, (b) cost matrix and optimal path (red line) chosen by the algorithm, (c) cost cumulative matrix, (d) correlation after alignment algorithm. The root-mean square errors (RMSE) prior and after temporal alignment are also reported.

- Boundary conditions: $w_{1}=(1,1)$ and $w_{L}=\left(N_{S}, N_{S}\right)$;

- Monotonicity condition: $t_{M R I_{1}} \leq t_{M R I_{2}} \leq \ldots \leq t_{M R I_{L}}$ and $t_{U S_{1}} \leq t_{U S_{2}} \leq \ldots \leq t_{U S_{L}}$;

- Step size conditions: $\left(w_{l+1}-w_{l}\right) \in(0,1),(1,0),(1,1)$ for $l=[1, \ldots, L-1]$.

The boundary condition establishes the alignment of the first and the last frame of $r_{U S}$ respectively with the first and last frame of $r_{M R I}$. In this way, the DTW algorithm will be applied to the two entire sequences. The monotonicity condition is necessary to maintain the chronological frame order in the two sequences. Finally, the step size condition expresses a continuity condition: no frame can be omitted and the state should always result in one-step time skip in, at least, one sequence.

The optimal temporal alignment is given as an optimal path in $c\left(t_{M R I}, t_{U S}\right)$. Intuitively, such an optimal alignment runs along a "valley" within the cost matrix $c\left(t_{M R I}, t_{U S}\right)$ and can be found through the estimation of a cumulative cost matrix $D\left(t_{M R I}, t_{U S}\right)$ recursively defined from $c\left(t_{M R I}, t_{U S}\right)$. Using as a start point the condition $D\left(t_{M R I_{1}}, t_{U S_{1}}\right)=c\left(t_{M R I_{1}}, t_{U S_{1}}\right)$ is possible to define $D$ as:

$$
D\left(t_{M R I_{l}}, t_{U S_{l}}\right)=c\left(t_{M R I_{1}}, t_{U S_{1}}\right)+\min \left(D\left(t_{M R I_{l-1}}, t_{U S_{l}}\right), D\left(t_{M R I_{l}}, t_{U S_{l-1}}\right), D\left(t_{M R I_{l-1}}, t_{U S_{l-1}}\right)\right) ;
$$


As shown in Figure 2c, the matrix $D$ lead to the optimal path $w$ that allows the best alignment between the two sequences. More details on the estimation of the DTW optimal path are omitted in the current manuscript, and the reader referred to Muller et al. ${ }^{16}$ for further details. Once the optimal path has been defined, the matching between $r_{M R I}$ and $r_{U S}$ can be used to temporally align the underlying MRI and US sequences from where the correlation-based signals $r$ were generated.

\section{EXPERIMENTS}

The proposed framework was evaluated using image data from 98 patients, which have undergone both 3D+t MRI and 3D-US trans-thoracic scans. The data used in the present study has been acquired at the enrollment of patients in a large ongoing multi-center clinical study (DOPPLER-CIP). ${ }^{17}$

We carried an initial validation experiment in order to verify if the information within the correlation-based signal $r$ was indeed consistent with the underlying temporal location of the key moments in the cardiac cycle. To this end, we have evaluated the accuracy of automatic end-systolic frame detection, estimated as the minimum of the correlation-based signal $r$, versus the end-systolic instant manually annotated by the clinical expert. The correspondence of the aligned sequences was visually confirmed and quantitatively assessed by calculating the root mean square error (RMSE) between the aligned correlation-based signals, $r_{M R I}$ and $r_{U S}$, from the respective MRI and RT3DE sequences. In order to provide a baseline for comparison, the RMSE was equally estimated prior to the temporal re-alignment of both signals.

\section{RESULTS}

Table 1 summarizes the results concerning the identification of the end-systolic frame detection on MRI and US sequences, obtained comparing the minimum of the correlation sequence with expert identification.

An average RMSE of $0.035 \pm 0.025$ was found after temporal synchronization of both $r_{M R I}$ and $r_{U S}$, which is statistically significantly lower than the $0.14 \pm 0.05$ estimated prior to the temporal re-alignment. An example of the values for the RMSE prior and after temporal alignment is given in Figure 2a and 2d, respectively. The average computational time necessary to compute the image-based correlation signal was $0.0421 \pm 0.0085 \mathrm{~s}$ for each 3D US frame and $0.0037 \pm 0.0022 s$ for each MRI frame, while the mean computational time necessary to apply DTW algorithm was $0.0088 \pm 0.0003 s$.

Table 1: End-systolic (ES) frame detection as the minimum of the correlation based signal $r$. Both absolute and relative ES detection errors (mean \pm standard deviation) were used as metric. $N_{\text {frames }}$ is the total number of frames in each sequence.

\begin{tabular}{ccc}
\hline & $\begin{array}{c}\text { Absolute ES detection error (frames) } \\
\left|f_{E D}-\min (r)\right|\end{array}$ & $\begin{array}{c}\text { Relative ES detection error (\%) } \\
\left|\left(f_{E D}-\min (r)\right) / N_{\text {frames }}\right|\end{array}$ \\
\hline MRI & $0.55 \pm 0.54$ & $1.60 \pm 1.89$ \\
US & $1.29 \pm 1.73$ & $4.03 \pm 4.23$ \\
\hline
\end{tabular}

\section{DISCUSSION}

In this paper, we presented an image-based framework for temporal synchronization of multimodality cardiac $4 \mathrm{D}$ image sequences. In first instance, we define the correlation curve employing exclusively image features. Several authors in previous works ${ }^{10,14}$ have noticed a relationship between this curve and the LV volume over time for US and MRI data, respectively. The pattern followed by the curves defined is similar: initially, there is a gradual decrease of the correlation coefficients during LV contraction, ending as a minimum near the endsystolic instant. After systole, the coefficients begin progressively to increase during LV relaxation. At the end of the ventricular diastole, the value of the correlation reaches the initial value and the pattern restarts. We apply this algorithm in 4D MRI and RT3DE to have an image-based, temporally continuous descriptor of the 
cardiac deformation, which can be used to retrieve temporal landmarks for temporal alignment. The result is a fast and reliable framework to identify these features, whose outcome is a starting point for the application of the DTW algorithm. The precise end-systolic frame detection in MRI and US 4D sequences was the chosen target in order to properly verify the consistency of the correlation-based signal with the underlying cardiac deformation. Following the indication of Kachenoura et al. ${ }^{14}$ this particular frame should be identified as the minimum of the correlation function. As shown in Table 1, the algorithm is more reliable in MRI than in US images, in which the end-systolic frame detection was, in some cases, missed by 3 frames. Different reasons might exist for these sub-optimal detections. Above all, the RT3DE sequences show a lower image quality, compared to MRI. The introduction of unexpected interferences in the field of view (body tissues) as much as random noise, which usually affects this image modality, induces a decorrelation responsible for the misdetection of the end-systolic frame. Moreover, the motion of the heart valves, clearly depicted in US while barely visible on MR imaging, represents another undesired source of decorrelation. Note that the algorithm could be improved by pre-processing each 3D image through a spatial smoothing filter. Such solution would decrease the random noise's influence, at the cost of additional computational load.

While previous works focused on the use of the correlation-based signal $r$ to detect temporal landmarks, such as the end-systolic instant, we rather use it as a temporally continuous descriptor of the underlying cardiac deformation process. We thus employed the DTW algorithm to find the optimal superposition between the entire image-based correlation curves. The algorithm warps the two sequences to minimize the differences between them, resulting in an overlap of key stages of the cardiac cycle. The outcomes, reported in Table 1, show a noticeable reduction concerning the curve's difference RMSE, thus pointing towards potential improvements in temporal alignment of the underlying cardiac deformation. Nevertheless, the overall footages alignment could exhibit some imperfection, since it relies on the cross-correlation function, whose computation could be impaired by images artifact. In precedent works, as Perperidis et al., ${ }^{10}$ a $4 \mathrm{D}$ transformation model is suggested for spatial and temporal alignment between two cardiac datasets. The (local) temporal synchronization relies on the identification of specific temporal points of the cardiac cycle. The alignment occurs, initially, by temporally overlapping these points and, subsequently, warping the two curves in the space between them. Therefore, the main novelty introduced by this work is the employment of the entire correlation curves in order to align the two sequences. This means that not only the relevant points of both sequences are overlapped, but also all the curves trend are taken into account for optimal alignment purpose. Once the algorithm has computed the optimal frame succession, the real alignment could occur, transferring the new frame chronology to the 4D sequences. Moreover, the reduced computational burden of the proposed approach allows its real-time application while intra-operatively scanning the patient. The 3D-US sequences mentioned on experiments section were acquired in a trans-thoracic apical view altough, in fact, the realization of whole this project was conducted aiming to temporally register pre-operative cine-MRI with 4D intra-operative transesophageal echocardiography (TEE) images. Even so, since the TEE usually exceeds trans-thoracic ultrasound image quality, whilst keeping the same intensity behavior over time, the key findings of the study will likely translate to consistent algorithm behavior in both modalities.

The independence of the method towards different modalities remains still an assumption that require further and thorough examination. Nonetheless, the results obtained in this initial approach are promising, giving credence to the abovementioned assumption, while opening the possibilities for further developments.

It must be stressed that an overall quantitative validation of the temporal alignment is not possible since there is no absolute ground truth. Alternatively, we performed a qualitative evaluation of the result analyzing the synchronized data, highlighting the end-systolic frames. Upon visual inspection, it was found that the temporal matching of the end-systolic moment could be improved after the proposed temporal alignment. Therefore, we have visually validated the method and its applicability in temporal synchronization of multimodal cardiac 4D sequences. The application of the framework here described provides the opportunity to apply a subsequent real-time spatial registration between images acquired in the same stage of the cardiac cycle, improving the fusion of pre-operative and intra-operative sequences, mandatory for image-guided applications. 


\section{CONCLUSIONS}

In this paper, we present a method to temporally synchronize multimodal cardiac sequences through the use of robust, image-derived signals which encode the temporal key moments of the cardiac cycle. The proposed method was found to be accurate and computationally fast, allowing the synchronization of MRI and RT3DE sequences of the same patient in intra-operative scenarios. Nonetheless, the method remains generic and could be applied to other modalities, such as computed tomography or single-photon emission computed tomography, or to inter-patient temporal alignment, which could be then employed on the construction of temporally consistent statistical shape and motion models. We did not verify our framework on intra-operative scenarios in which the computational time is a crucial factor. Moreover, the presence of surgical tools in the image might introduce an unforeseen variation on the correlation curve. For this reason, future work will involve further validation on intra-operative TEE images, verifying both the robustness of the method and its real-time applicability.

\section{ACKNOWLEDGMENTS}

This work was supported by FCT - Fundação para a Ciência e Tecnologia, Portugal, in the scope of the project EXPL/BBB-BMD/2473/2013 and PhD grant SFRH/BD/93443/2013. The authors would also like to acknowledge the DOPPLER-CIP consortium (EU/FP7 framework program, grant number 223615) for giving access to the data. D. Barbosa would also like to acknowledge the kind support of the Fundação Luso-Americana para o Desenvolvimento (FLAD), which has funded the travel costs for participation at SPIE Medical Imaging 2015.

\section{REFERENCES}

1. V. Mor-Avi, L. Sugeng, and R. M. Lang, "Real-time 3-dimensional echocardiography an integral component of the routine echocardiographic examination in adult patients?," Circulation 119(2), pp. 314-329, 2009.

2. J. W. Cannon, J. A. Stoll, I. S. Salgo, H. B. Knowles, R. D. Howe, P. E. Dupont, G. R. Marx, and P. J. del Nido, "Real-time three-dimensional ultrasound for guiding surgical tasks," Computer aided surgery 8(2), pp. 82-90, 2003.

3. J. Balzer, H. Kühl, T. Rassaf, R. Hoffmann, P. Schauerte, M. Kelm, and A. Franke, "Real-time transesophageal three-dimensional echocardiography for guidance of percutaneous cardiac interventions: first experience," Clinical Research in Cardiology 97(9), pp. 565-574, 2008.

4. K. Cleary and T. M. Peters, "Image-guided interventions: technology review and clinical applications," Annual review of biomedical engineering 12, pp. 119-142, 2010.

5. T. Makela, P. Clarysse, O. Sipila, N. Pauna, Q. C. Pham, T. Katila, and I. E. Magnin, "A review of cardiac image registration methods," Medical Imaging, IEEE Transactions on 21(9), pp. 1011-1021, 2002.

6. J.-M. Peyrat, H. Delingette, M. Sermesant, C. Xu, and N. Ayache, "Registration of 4D cardiac CT sequences under trajectory constraints with multichannel diffeomorphic demons," Medical Imaging, IEEE Transactions on 29(7), pp. 1351-1368, 2010.

7. X. Huang, J. Ren, G. Guiraudon, D. Boughner, and T. M. Peters, "Rapid dynamic image registration of the beating heart for diagnosis and surgical navigation," Medical Imaging, IEEE Transactions on 28(11), pp. 1802-1814, 2009.

8. G. Van Der Hoeven, P. Clerens, J. Donders, J. Beneken, and J. Vonk, "A study of systolic time intervals during uninterrupted exercise.," British heart journal 39(3), pp. 242-254, 1977.

9. R. Shekhar, V. Zagrodsky, M. J. Garcia, and J. D. Thomas, "Registration of real-time 3-D ultrasound images of the heart for novel 3-D stress echocardiography," Medical Imaging, IEEE Transactions on 23(9), pp. 1141-1149, 2004.

10. D. Perperidis, R. H. Mohiaddin, and D. Rueckert, "Spatio-temporal free-form registration of cardiac MR image sequences," Medical image analysis 9(5), pp. 441-456, 2005.

11. W. Zhang, J. M. Brady, H. Becher, and J. A. Noble, "Spatio-temporal (2D+ T) non-rigid registration of realtime 3D echocardiography and cardiovascular MR image sequences," Physics in medicine and biology 56(5), p. 1341, 2011.

12. K. Wang, T. Gasser, et al., "Alignment of curves by dynamic time warping," The Annals of Statistics 25(3), pp. 1251-1276, 1997. 
13. B. Raghavendra, D. Bera, A. S. Bopardikar, and R. Narayanan, "Cardiac arrhythmia detection using dynamic time warping of ECG beats in e-healthcare systems," in World of Wireless, Mobile and Multimedia Networks (WoWMoM), 2011 IEEE International Symposium on a, pp. 1-6, IEEE, 2011.

14. N. Kachenoura, A. Delouche, A. Herment, F. Frouin, and B. Diebold, "Automatic detection of end systole within a sequence of left ventricular echocardiographic images using autocorrelation and mitral valve motion detection," in Engineering in Medicine and Biology Society, 200\%. EMBS 200\%. 29th Annual International Conference of the IEEE, pp. 4504-4507, IEEE, 2007.

15. M. Afshin, I. B. Ayed, A. Islam, A. Goela, T. M. Peters, and S. Li, "Global assessment of cardiac function using image statistics in MRI," in Medical Image Computing and Computer-Assisted Intervention-MICCAI 2012, pp. 535-543, Springer, 2012.

16. M. Müller, "Dynamic time warping," Information retrieval for music and motion, pp. 69-84, 2007.

17. F. Rademakers, J. Engvall, T. Edvardsen, M. Monaghan, R. Sicari, E. Nagel, J. Zamorano, H. Ukkonen, T. Ebbers, V. Di Bello, et al., "Determining optimal noninvasive parameters for the prediction of left ventricular remodeling in chronic ischemic patients," Scandinavian Cardiovascular Journal 47(6), pp. 329$334,2013$. 